\title{
Infection by Candidatus Liberibacter solanacearum' haplotypes A and B in Solanum lycopersicum 'Moneymaker'
}

Azucena Mendoza-Herrera, Department of Entomology, Julien Levy ${ }^{\dagger}$ and Kyle Harrison, Department of Horticultural Sciences, and Jianxiu Yao, Freddy Ibanez, and Cecilia Tamborindeguy, ${ }^{\dagger}$ Department of Entomology, Texas A\&M University, College Station 77843

\begin{abstract}
'Candidatus Liberibacter solanacearum' is a plant pathogen associated with diseases affecting several crops of the Solanaceae and Apiaceae families. Two ' $\mathrm{Ca}$. L. solanacearum' haplotypes (LsoA and LsoB) infect solanaceous crops in North America and are transmitted by the tomato psyllid Bactericera cockerelli. Although both ' $C a$. L. solanacearum' haplotypes cause zebra chip in potato, the diseases associated with each haplotype in tomato (Solanum lycopersicum) have not been described. 'Ca. L. solanacearum'-infected tomato plants exhibit symptoms resembling those of permanent yellowing disease (known in Mexico as "permanente del tomate") and sometimes called psyllid yellows. In this study, the symptoms associated with each ' $\mathrm{Ca}$. L. solanacearum'

haplotype in tomato were compared, and the bacterial abundance in different nodes of the plants was measured by quantitative polymerase chain reaction. Surprisingly, both plant phenotype and bacterium distribution were different between LsoA- and LsoB-infected plants. Plants infected with LsoB died prematurely, whereas those infected with LsoA did not. Across the measured time points, LsoB abundance in infected plants was consistent with previous reports describing a sink to source gradient, while such gradient was only observed in LsoA-infected plants early after infection. This is the first report describing the differences in symptoms in tomato associated with two ' $\mathrm{Ca}$. L. solanacearum' haplotypes, LsoA and LsoB.
\end{abstract}

'Candidatus Liberibacter solanacearum' is a gram-negative bacterium (Liefting et al. 2008) and infects plants worldwide. Presently, five ' $\mathrm{Ca}$. L. solanacearum' haplotypes (LsoA, LsoB, LsoC, LsoD, and LsoE) have been identified (Glynn et al. 2012; Lin et al. 2012; Nelson et al. 2011). LsoA and LsoB are two haplotypes transmitted by tomato psyllid (also known as potato psyllid) Bactericera cockerelli (Hemiptera: Triozidae), and infect solanaceous plants in the Americas and New Zealand (Liefting et al. 2008, 2009; N. Wang et al. 2017). In contrast, LsoC, LsoD, and LsoE are transmitted by carrot psyllids Trioza apicalis and B. trigonica and infect apiaceous crops in Europe, North Africa, and the Middle East (Alfaro-Fernández et al. 2012; Munyaneza et al. 2010; NPPO 2017; Tahzima et al. 2014; Teresani et al. 2014).

Zebra chip, a potato disease associated with ' $\mathrm{Ca}$. L. solanacearum', was first described in Mexico in 1994 and reported in the United States in 2000 (Secor and Rivera-Varas 2004). Today, it has become a major disease of potato in the western half of the United States, Mexico, Central America, and New Zealand, and is responsible for millions of dollars in annual losses to the potato industry (Crosslin et al. 2010). Several aspects of this disease have been studied in detail. For instance, typical aerial symptoms associated with zebra chip are yellowing, mild purpling, curling, and scorching of

Current address of J. Yao: College of Veterinary Medicine, Kansas State University, Manhattan 66506

Current address of F. Ibanez: Entomology and Nematology Department, Citrus Research and Education Center, University of Florida, Lake Alfred 33850.

${ }^{\dagger}$ Corresponding authors: J. Levy, E-mail: julienlevy@tamu.edu; and C. Tamborindeguy, E-mail: ctamborindeguy@tamu.edu

Funding: This work was supported by United States Department of Agriculture-National Institute for Food and Agriculture grants 2012-67013-19431 and 2017-67013-26564 and by the Texas A\&M AgriLife Research Insect Vector Diseases Grant Program Vector Initiative.

*The $\boldsymbol{e}$-Xtra logo stands for "electronic extra" and indicates that two supplementary figures and one supplementary movie are published online.

Accepted for publication 30 March 2018.

C 2018 The American Phytopathological Society leaves (Crosslin et al. 2010). Foliar symptoms are accompanied by inhibition of the root development, which might account for the symptoms associated with nutritional deficiency (Spann and Schumann 2009). Infected tubers exhibit internal necrosis and display a characteristic striped pattern after being fried as chips (Munyaneza et al. 2007), which makes them unmarketable. Other symptoms include the reduction of plant vigor and early plant death.

The diseases associated with ' $\mathrm{Ca}$. L. solanacearum' in other solanaceous crops have not been studied in such depth. In tomato, symptoms associated with ' $\mathrm{Ca}$. L. solanacearum' infection include spiky apical leaves, chlorosis and mottling of leaves, plant stunting, and fruit deformation (Liefting et al. 2009). However, a later study investigating ' $\mathrm{Ca}$. L. solanacearum' infection in Moneymaker and Roma tomato found that some tomato plants that tested positive for ' $\mathrm{Ca}$. L. solanacearum' infection did not develop foliar symptoms, whereas other plants developed typical symptoms including plant death $(\mathrm{Li}$ et al. 2013). In symptomatic plants, symptoms started to develop within 4 to 6 weeks after infection. Previously, we have shown that, although ' $C a$. L. solanacearum' can be detected consistently in the upper, middle, and lower tier of tomato plants 5 to 6 weeks after infection, leaf curling and yellowing first developed in the upper leaves 8 weeks after infection (Levy et al. 2011). Today, we know that the ' $C a$. L. solanacearum'-infected colony used in that study harbored both LsoA and LsoB haplotypes.

Although the existence of two ' $\mathrm{Ca}$. L. solanacearum' haplotypes infecting solanaceous crops was discovered in 2009 (Wen et al. 2009), the epidemiological consequences of the ' $\mathrm{Ca}$. L. solanacearum' genetic diversity and the pathogenicity of each ' $\mathrm{Ca}$. L. solanacearum' haplotype in vectors and plant hosts remain unknown. In the United States, LsoA and LsoB can be found alone or coinfecting plants and vectors. Both ' $\mathrm{Ca}$. L. solanacearum' haplotypes cause zebra chip and result in the premature death of potato plants but spatial and temporal distribution analyses of each ' $\mathrm{Ca}$. L. solanacearum' haplotype in the United States showed that LsoB infection could result in more severe symptoms than LsoA infection (Wen et al. 2013).

In this study, we investigated the effect of LsoA and LsoB infection in tomato plants. In particular, we wanted to evaluate whether both ' $\mathrm{Ca}$. L. solanacearum' haplotypes can infect tomato, and the symptoms associated with each ' $\mathrm{Ca}$. L. solanacearum' haplotype in this plant species. Furthermore, we wanted to determine whether differences in pathogenicity or bacterial distribution exist between the haplotypes in association with tomato plants. Our data will pave the way to understanding the differences in LsoA 
and LsoB pathogenicity and can be used to better adapt programs to control 'Ca. L. solanacearum'-related diseases or to identify host plant resistance (Levy and Tamborindeguy 2014; Lévy et al. 2015).

\section{Materials and Methods}

Insects. A ' $C a$. L. solanacearum'-free B. cockerelli colony was obtained in 2013 from Dr. Henne, Texas A\&M AgriLife Research at Weslaco, and was reared on tomato plants in insect-proof cages (Bioquip Inc., Compton, CA) at room temperature with a photoperiod of $16 \mathrm{~h}$ of light and $8 \mathrm{~h}$ of darkness.

B. cockerelli colonies carrying either LsoA or LsoB were obtained by rearing insects from the ' $\mathrm{Ca}$. L. solanacearum'-free colony on LsoA- and LsoB-infected tomato plants, respectively (Yao et al. 2016). These colonies are henceforth referred to as LsoA and LsoB colonies, respectively. Diagnostic polymerase chain reaction (PCR) analyses to detect the presence of ' $\mathrm{Ca}$. L. solanacearum' and to identify the ' $C a$. L. solanacearum' haplotype were performed for all $B$. cockerelli colonies, as described previously (Nachappa et al. 2011, 2014). Presently, there are four different psyllid haplotypes in the United States; the psyllids used in this study are from the Northwestern haplotype.

Plant source and plant infection. All experiments were performed using tomato plants (Solanum lycopersicum L. 'Moneymaker'; Thompson \& Morgan Inc., Jackson, NJ). Plants were grown from seed in pots with Sun Gro Sunshine LP5 mix (Bellevue, WA) and fertilized twice a week with Miracle-Gro Water-Soluble Tomato Plant Food at the label rate (18-18-21 NPK; Scotts Miracle-Gro Company, Marysville, OH). Four-week-old seedlings were transferred to 10 -by-10-cm pots, individually. All experiments were performed 1 week after transplanting, when plants had four fully expanded leaves.

To obtain LsoA- and LsoB-infected plants, two adult male psyllids from the LsoA or the LsoB colonies were caged for a week in organza bags on the bottom-most leaf of each plant (Supplementary Fig. S1). After a week, the insects were removed by cutting the leaf with the organza bag. The day the insects were removed was considered day 1 after infestation (it was also considered day 1 postinfection for plants infested with LsoA or LsoB psyllids). There were two control treatments: ' $C a$. L. solanacearum'-free treatment, in which plants were infested for a week with two psyllids from the ' $\mathrm{Ca}$. L. solanacearum'-free colony, and the control, in which plants were not infested with psyllids. Plants in the ' $\mathrm{Ca}$. L. solanacearum'-free treatment had undetectable levels of ' $\mathrm{Ca}$. L. solanacearum', similar to control plants. Those plants were included to control for potential symptoms caused by psyllid feeding during inoculation. All plants were kept at room temperature with a photoperiod of $16 \mathrm{~h}$ of light and $8 \mathrm{~h}$ of darkness until the end of the experiment.

Symptom progression. Plants were observed weekly to record symptoms associated with each ' $C a$. L. solanacearum' haplotype. To compare the progression of symptoms associated with each haplotype, one plant from each treatment was placed on a wire shelf and photographed every 2 days (Supplementary Movie).

Progression of ' $\mathrm{Ca}$. L. solanacearum' abundance on toptier leaves. Leaf samples were collected from the top-tier leaves weekly following infestation from week 1 to 5 . Samples were collected also at week 8 , which represents a time point at which LsoB-infected plants displayed advanced infection symptoms and were dying, whereas LsoA-infected plants only displayed stunting and foliar symptoms. DNA was extracted from these samples as previously described (Levy et al. 2011). This DNA was used as a PCR template to verify the presence of ' $\mathrm{Ca}$. L. solanacearum' and the ' $\mathrm{Ca}$. L. solanacearum' haplotype at weeks 4, 5, and 8 using the LsoF/OI2 and SSR1 primers, respectively (Li et al. 2009, Lin et al. 2012). Each week postinoculation, relative ' $\mathrm{Ca}$. L. solanacearum' abundance in tomato plants was quantified by quantitative PCR (qPCR) using the LsoF and HLBR primer set, as described by Levy et al. (2011). This experiment was repeated three times, each time using 5 plants per treatment (45 plants total). ' $C a$. L. solanacearum' abundance was measured in four plants per infected treatment per replicate (i.e., LsoA-infected and LsoB-infected tomato) and from two plants from each control treatment (' $\mathrm{Ca}$. L. solanacearum'-free and control tomato).

' $\mathrm{Ca}$. L. solanacearum' distribution and abundance in different nodes of tomato plants. Another experiment evaluated ' $\mathrm{C} a$. L. solanacearum' abundance in different tomato plant nodes at weeks 3,5 , and 7 postinoculation. Counting from the apical meristem, tissues were collected from the base of every odd-numbered leaf. DNA was extracted from 206 plant tissue samples previously infected with LsoA (6 plants and 105 samples) or LsoB (6 plants and 101 samples); these tissue samples corresponded to three time points and each of 7 to 14 tomato nodes, depending on the plant development. Root tissues were also collected at each time point from these plants and processed as described for leaves. As previously described, DNA was extracted and ' $\mathrm{Ca}$. L. solanacearum' abundance was quantified by qPCR.

'Ca. L. solanacearum' quantification. To quantify ' $\mathrm{Ca}$. L. solanacearum' abundance in infected tomato plants, qPCR was performed with the following amplification protocol: 40 cycles of $95^{\circ} \mathrm{C}$ for $15 \mathrm{~s}$ and $60^{\circ} \mathrm{C}$ for $30 \mathrm{~s}$ using an ABI 7900HT Fast Thermocycler (Applied Biosystems, Waltham, MA). Each reaction contained $30 \mathrm{ng}$ of DNA, $0.25 \mu \mathrm{M}$ each primer, $5 \mu \mathrm{l}$ of SYBR Green Master Mix (SensiFAST SYBR; Bioline USA, Taunton, MA), and $3 \mu l$ of water. Reactions for all samples were performed in duplicate, with positive and negative controls in each run. The mean threshold cycles values and standard deviation were calculated.

Data analysis. The ' $C a$. L. solanacearum' standard curve was used to quantify ' $\mathrm{Ca}$. L. solanacearum' in tomato plants, as was described by Levy et al. (2011). The data obtained after ' $\mathrm{Ca}$. L. solanacearum' quantification using the standard curve method was logtransformed. All statistical analyses were conducted using JMP, version 13 (SAS Institute Inc., Cary, NC). For the experiment tracking ' $C a$. L. solanacearum' abundance in top-tier leaves, a standard least-squares model was built to test the effect leverage of ' $\mathrm{Ca}$. L. solanacearum' abundance to infection treatment (i.e., LsoA-infected, LsoB-infected, and uninfected), week number, and their combination while individual plants were treated as a repeated measure. For the experiment tracking ' $\mathrm{Ca}$. L. solanacearum' abundance in whole tomato plants, a standard least-squares model was built to test the response of ' $\mathrm{Ca}$. L. solanacearum' abundance to the full-factorial of three variables: (i) node number counting from the apical meristem, (ii) week number, and (iii) ' $C a$. L. solanacearum' haplotype. Because the number of replicates per treatment was limited in this experiment, the singular value decomposition method of multiple imputation was performed to replace the missing values from individual plants in a given week or node. Furthermore, a goodness-of-fit analysis was performed between ' $\mathrm{Ca}$. L. solanacearum' abundance and node number for each combination of ' $\mathrm{Ca}$. L. solanacearum' haplotype and week number (six models total). A factorial analysis of variance was conducted to compare the effects of ' $\mathrm{Ca}$. L. solanacearum' haplotype, week tested (weeks 3, 5, and 7), and their interaction on ' $\mathrm{Ca}$. L. solanacearum' abundance in the root of infected plants. Pairwise comparisons between the ' $\mathrm{Ca}$. L. solanacearum' abundance per week sampled were performed using Tukey's honestly significant difference test.

\section{Results}

Progression of symptoms associated with ' $\mathrm{Ca}$. L. solanacearum' infection. Differential symptomatology associated with LsoA and LsoB infection developed in ' $\mathrm{Ca}$. L. solanacearum'infected tomato. During the first 3 weeks following infestation, no distinguishable symptoms developed in the ' $\mathrm{Ca}$. L. solanacearum'-infected plants and there were no differences in plant growth. However, later, a reduction in plant growth was observed in the infected plants compared with the control plants. From week 3 to week 5, differences in the size of the new leaves on the top of the infected plants developed. New leaves in the LsoB-infected plants were smaller (Fig. 1A) and had some discoloration. Meanwhile, in LsoA-infected plants, the shape and the color of the leaves was more similar to the uninfected plants. Additionally, new leaves 
started developing from the bottom-tier axillary meristems in the LsoA-infected plants (Fig. 1B).

After week 5, LsoB-infected plants showed stunting, curling, yellowing, and some necrotic areas compared with the noninfected plants or even with the LsoA-infected plants (Fig. 1). The LsoAinfected plants were stunted compared with the uninfected plants. By week 6, the LsoB-infected plants had stopped growing, the younger and older leaves were dying, and necrotic areas continued to develop, while the LsoA-infected plants continued to develop new leaves and continued to grow but remained shorter than noninfected plants. As time progressed, necrosis spread in LsoB-infected plants while no new leaves developed and the plants wilted. By week 8, LsoB-infected plants were dead or dying. LsoA-infected plants remained alive and green, even though some discoloration persisted; they continued to grow but were stunted compared with the noninfected plants (Table 1; Supplementary Fig. S2).

' $\mathrm{Ca}$. L. solanacearum' abundance progression based on toptier leaves. ' $C a$. L. solanacearum' abundance in tomato plant was measured from weeks 1 to 5 and at week 8 following infestation in uninfected, LsoA-infected, and LsoB-infected plants. There was a whole-model effect in the standard least-squares model of ' $\mathrm{Ca}$. L. solanacearum' abundance across weeks $\left(F_{5}, 215=141.3231, P<\right.$ 0.0001). Effect tests indicated that treatment (sum of squares $62.76373, F$ ratio $=66.2197, P<0.0001$ ), week (sum of squares $=$ $221.23400, F$ ratio $=233.4159, P<0.0001$ ), and their combination (sum of squares $=50.87098, F$ ratio $=107.3442, P<0.0001)$ each had significant effects on ' $\mathrm{Ca}$. L. solanacearum' abundance. Parameter estimates of the standard least-squares model also indicated significant differences among treatment and week, consistent with the effect tests. Parameter estimates also indicated that differences in LsoA and LsoB abundance could be detected after week 3.83333 (Table 2; note that LsoA and LsoB abundance intersect at approximately 3.8888 weeks in Figure 2). Specifically, abundance of LsoA initially increased but leveled off by week 3, whereas LsoB abundance increased throughout the experiment (Fig. 2). By week 2, LsoA- and LsoB-infected plants had detectable levels of ' $\mathrm{Ca}$. L. solanacearum', whereas ' $\mathrm{Ca}$. L. solanacearum' levels remained undetectable in the control plants at all the tested time points. Also, it is noteworthy that LsoA abundance seemed to increase faster than LsoB abundance up to week 3 .

' $\mathrm{Ca}$. L. solanacearum' distribution in tomato plants. To further validate the results obtained in the prior experiment (specifically, the decrease in LsoA abundance after week 3), 'Ca. L. solanacearum' was quantified in different nodes of LsoA- and LsoB-infected plants at weeks 3, 5, and 7 after infection. Depending on the plant development at the sampling time, between 7 and 14 nodes were sampled. There was a whole-model effect in the standard least-squares model of LsoA and LsoB abundance across nodes and weeks $\left(\mathrm{F}_{7,198}=\right.$ 15.67, $P<0.0001$ ). Effect tests indicated that week (sum of squares $=$ $28.426193, F$ ratio $=30.5038, P<0.0001$ ), haplotype (sum of squares $=10.00585, F$ ratio $=10.7369, P=0.0012$ ), and node ( sum of squares $=4.1288, F$ ratio $=4.4306, P=0.0366)$ had significant effects on ' $\mathrm{Ca}$. L. solanacearum' abundance, and all interactions among these factors were also significant (Table 3).
Specifically, for weeks 5 and 7, ' $\mathrm{Ca}$. L. solanacearum' abundance in nodes higher up on the LsoA-infected plants (node $<5$ ) was significantly different from LsoB-infected plants (Table 4). Parameter estimates for the standard least-squares model also showed that plants infected with different ' $\mathrm{Ca}$. L. solanacearum' haplotypes had a significantly different ' $\mathrm{Ca}$. L. solanacearum' abundance that could be detected after week 5 (regression coefficient 5.05825) and in top-tier nodes (node < 5.06311) (Table 4). The goodness-of-fit models supported these findings (Fig. 3). In weeks 5 and 7, LsoA was less abundant in the upper nodes of ' $\mathrm{Ca}$. L. solanacearum'infected tomato plants compared with LsoB (Fig. 3). LsoA abundance in weeks 5 and 7 were the only conditions that generated an exponential trend line with an intercept $<1.0$; in other words, that ' $\mathrm{Ca}$. L. solanacearum' abundance in the top-tier leaves was below detection threshold.

Overall, in LsoB-infected plants, a ' $\mathrm{Ca}$. L. solanacearum'-abundance gradient was observed at each time point, with higher ' $\mathrm{Ca}$. L. solanacearum' levels in the younger leaves. A similar ' $C a$. L. solanacearum' gradient was measured in LsoA-infected plants at week 3 .

\section{A New leaves at week 5}
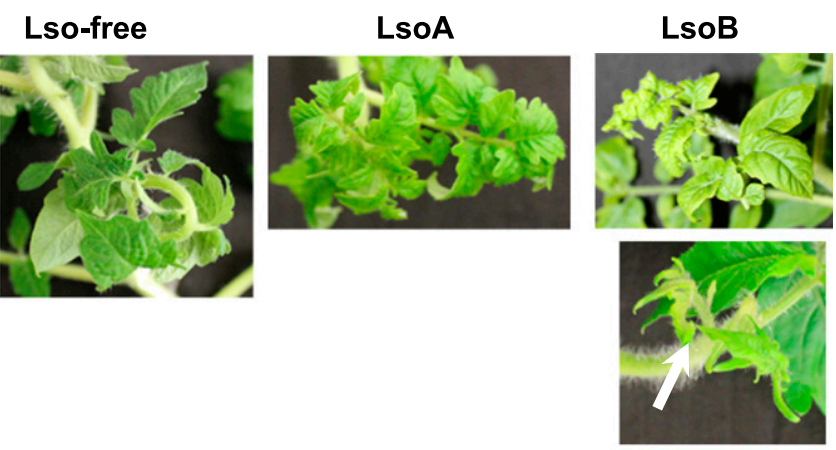

B Other Symptoms

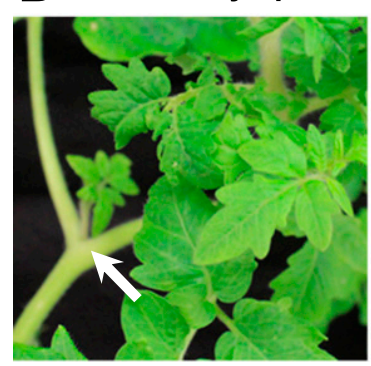

Axillary growth Lso A Week 6

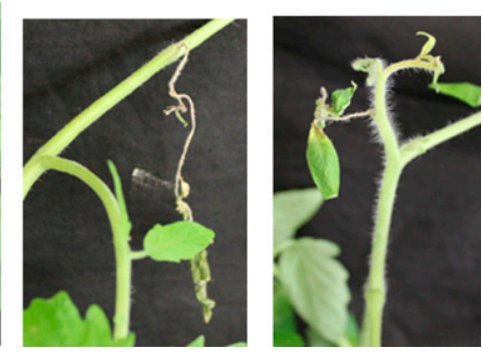

Necrosis

Lso B week 7
Fig. 1. Detail of key symptoms associated with 'Candidatus Liberibacter solanacearum' haplotypes LsoA and LsoB infection in tomato plants. A, New leaves in plants 5 weeks after infestation. B, Other symptoms in LsoA- and LsoB-infected plants.

Table 1. Timeframe for major symptom development following 'Candidatus Liberibacter solanacearum' haplotype LsoA and LsoB infection ${ }^{\mathrm{a}}$

\begin{tabular}{|c|c|c|c|c|c|}
\hline Haplotype & Week 1 to 3 & After week 3 & After week 5 & After week 6 & After week 8 \\
\hline LsoA & No symptoms & $\begin{array}{l}\text { New leaves on the apical } \\
\text { meristem are smaller; new } \\
\text { growth from axillary } \\
\text { meristems in the lower tier } \\
\text { of the plant }\end{array}$ & $\begin{array}{l}\text { Leaves from apical } \\
\text { meristem continue } \\
\text { developing but remain } \\
\text { smaller; plants are stunted }\end{array}$ & $\begin{array}{l}\text { Plants show some } \\
\text { discoloration }\end{array}$ & $\begin{array}{l}\text { The plant is alive and } \\
\text { growing but is smaller } \\
\text { than uninfected plants }\end{array}$ \\
\hline LsoB & No symptoms & $\begin{array}{l}\text { New leaves on the apical } \\
\text { meristem are smaller with } \\
\text { discoloration }\end{array}$ & $\begin{array}{l}\text { Leaves from apical } \\
\text { meristem stop developing; } \\
\text { plants are stunted }\end{array}$ & $\begin{array}{l}\text { Younger leaves display } \\
\text { curling and yellowing; } \\
\text { necrosis starts to develop }\end{array}$ & $\begin{array}{l}\text { The plant is dying or is } \\
\text { already dead }\end{array}$ \\
\hline Control & $\begin{array}{l}\text { No symptoms develop, } \\
\text { plants continue to grow }\end{array}$ & $\begin{array}{l}\text { No symptoms develop, } \\
\text { plants continue to grow }\end{array}$ & $\begin{array}{l}\text { No symptoms develop, } \\
\text { plants continue to grow }\end{array}$ & $\begin{array}{l}\text { No symptoms develop, } \\
\text { plants continue to grow }\end{array}$ & $\begin{array}{l}\text { No symptoms develop, } \\
\text { plants continue to grow }\end{array}$ \\
\hline
\end{tabular}

a Timing for symptom development may vary. 
Table 2. Parameter estimates for the standard least-squares model comparing the effect leverage of treatment ('Candidatus Liberibacter solanacearum' haplotype LsoA infection versus LsoB infection versus uninfected control) and week number on ' $\mathrm{Ca}$. L. solanacearum' abundance

\begin{tabular}{lccrc}
\hline Term & Estimate & $\begin{array}{c}\text { Standard } \\
\text { error }\end{array}$ & $\boldsymbol{t}$ Ratio & $\begin{array}{c}\text { Prob }> \\
|\boldsymbol{t}|^{\mathbf{a}}\end{array}$ \\
\hline Intercept & 0.5358099 & 0.09202 & 5.82 & $<\mathbf{0 . 0 0 0 1}$ \\
Control & -1.417364 & 0.066242 & -21.40 & $<\mathbf{0 . 0 0 0 1}$ \\
Haplotype A & 0.5364697 & 0.066242 & 8.10 & $<\mathbf{0 . 0 0 0 1}$ \\
Week & -0.214078 & 0.020663 & 10.36 & $<\mathbf{0 . 0 0 0 1}$ \\
Control $\times($ week = 3.83333) & -0.211635 & 0.029221 & -7.24 & $<\mathbf{0 . 0 0 0 1}$ \\
Haplotype A $\times($ week $=$ & -0.12051 & 0.029221 & -4.12 & $<\mathbf{0 . 0 0 0 1}$ \\
3.83333) & & & &
\end{tabular}

a Bold values indicate statistical significance.

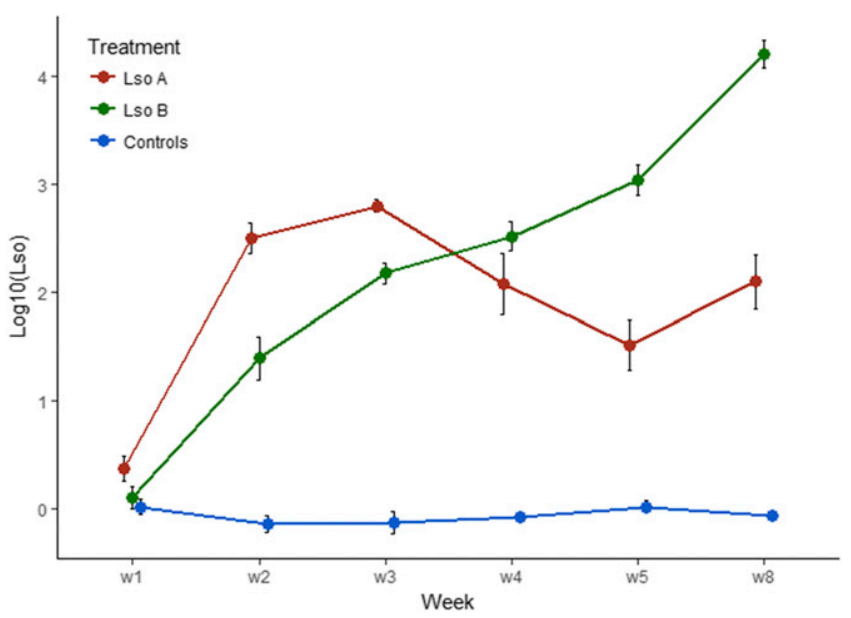

Fig. 2. Progression of 'Candidatus Liberibacter solanacearum' abundance in the top-tier leaves of tomato plants. Top leaves of plants were collected weekly up to 5 weeks following infestation; an additional sampling was performed at week 8 from controls and haplotype LsoA- and LsoB-infected plants. 'Ca. L. solanacearum' abundance was calculated using the standard curve method and log transformed (y-axis).

Table 3. Effect test results of the standard least-squares model comparing 'Candidatus Liberibacter solanacearum' abundance against week, 'Ca. L. solanacearum' haplotype, and node number

\begin{tabular}{lccr}
\hline Source & Sum of squares & $\boldsymbol{F}$ ratio & Prob $>\boldsymbol{F}$ a \\
\hline Week & 28.426193 & 30.5038 & $<\mathbf{0 . 0 0 0 1}$ \\
Haplotype & 10.005585 & 10.7369 & $\mathbf{0 . 0 0 1 2}$ \\
Node & 4.1288 & 4.4306 & $\mathbf{0 . 0 3 6 6}$ \\
Week $\times$ haplotype & 32.718314 & 35.1096 & $<\mathbf{0 . 0 0 0 1}$ \\
Week $\times$ node & 12.730363 & 13.7252 & $\mathbf{0 . 0 0 0 3}$ \\
Haplotype $\times$ node & 9.892159 & 10.6151 & $\mathbf{0 . 0 0 1 3}$ \\
Week $\times$ haplotype $\times$ node & 7.125589 & 7.6464 & $\mathbf{0 . 0 0 6 2}$ \\
\hline
\end{tabular}

a Bold values indicate statistical significance.
However, at weeks 5 and 7, higher ' $\mathrm{Ca}$. L. solanacearum' abundance was measured in the leaves from the plant middle tier compared with the bottom and top tiers. In weeks 5 and 7, the top-most leaves had undetectable levels of LsoA (Fig. 4).

Comparison of ' $\mathrm{Ca}$. L. solanacearum' abundance in the roots indicated that only the effect of week sampled was statistically significant $\left(F_{2,23}=23.2, P<0.001\right)$. The effect of ' $C a$. L. solanacearum' haplotype $\left(F_{1,23}=1.2, P=0.3\right)$ and of the interaction between ' $\mathrm{Ca}$. $\mathrm{L}$. solanacearum' haplotype and week sampled $\left(F_{2,23}=1.5, P=0.2\right)$ on ' $C a$. L. solanacearum' abundance in the roots were not statistically significant. ' $C a$. L. solanacearum' abundance in roots increased as the infection progressed (Fig. 5).

\section{Discussion}

Liberibacter bacteria are emerging as major threats to many crops worldwide (Haapalainen 2014; Tamborindeguy et al. 2017; N. Wang et al. 2017). However, our understanding of their interaction with plants and their psyllid vectors is still limited. At least two ' $\mathrm{Ca}$. $\mathrm{L}$. solanacearum' haplotypes (LsoA and LsoB) are associated with zebra chip and can infect and damage other solanaceous crops (Wen et al. 2009). Both haplotypes are currently present in the United States. The genetic diversity of ' $\mathrm{C} a$. L. solanacearum' can be used to better understand the role of different genes or metabolic pathways on disease progression in solanaceous plants. The objective of this study was to assess the effect of each ' $\mathrm{Ca}$. L. solanacearum' haplotype on the tomato host.

Infection with each ' $\mathrm{Ca}$. L. solanacearum' haplotype resulted in a reduction of plant growth after 3 weeks following infestation. Another common symptom associated with both haplotypes was the smaller size and spiky aspect of the new leaves on the apical growth. These symptoms were consistent with other reports of ' $\mathrm{Ca}$. L. solanacearum' infection in tomato (Liefting et al. 2009; RojasMartinez et al. 2016). Despite these common symptoms, there were major differences associated with LsoA and LsoB infection. Starting 4 weeks after infestation, leaves at the apical and basal extremities of the LsoB-infected plant started dying, and plants wilted and died prematurely. On the other hand, LsoA-infected plants had reduced apical growth, and new leaves grew from the axillary meristems in the plant middle and bottom tiers. More importantly, LsoA-infected plants did not die. In 2013, Li and coworkers reported that, following grafting of infected potato plants into tomato plants, half of these plants (two of four) did not develop symptoms and remained positive for ' $C a$. L. solanacearum' for 5 years, whereas the other half developed symptoms and died (Li et al. 2013). Although the authors did not test the ' $\mathrm{Ca}$. L. solanacearum' haplotype infecting the plants because they were unidentified at that time, these results are similar to those obtained in the present study, suggesting that the plants that developed symptoms and died were infected with $\mathrm{LsoB}$ whereas the ones that remained alive were infected with LsoA. The qualitative comparison of symptom development between LsoA- and LsoBinfected tomato plants suggested a greater severity of symptoms associated with LsoB infection, while early symptoms were observed at the same time for both haplotypes. The expression and severity of the symptoms associated with each ' $\mathrm{Ca}$. L. solanacearum' haplotype

Table 4. Parameter estimates for the standard least-squares model comparing the effect leverage of 'Candidatus Liberibacter solanacearum' haplotype, node, and week number on 'Ca. L. solanacearum' abundance

\begin{tabular}{|c|c|c|c|c|}
\hline Term & Estimate & Standard error & $t$ Ratio & Prob $>|t|^{\mathbf{a}}$ \\
\hline Intercept & 1.8946498 & 0.249981 & 7.58 & $<0.0001$ \\
\hline Week & 0.2362732 & 0.04278 & 5.52 & $<0.0001$ \\
\hline Haplotype & -0.224996 & 0.068665 & -3.28 & 0.0012 \\
\hline Node & -0.090047 & 0.04278 & -2.1 & 0.0366 \\
\hline$($ Week $=5.05825) \times$ haplotype $\mathrm{A}$ & -0.138555 & 0.023384 & -5.93 & $<0.0001$ \\
\hline$($ Week $=5.05825) \times($ node $=5.06311)$ & 0.0525602 & 0.014187 & 3.7 & 0.0003 \\
\hline Haplotype A $\times($ node $=5.06311)$ & 0.0761857 & 0.023384 & 3.26 & 0.0013 \\
\hline $\begin{array}{l}(\text { Week }=5.05825) \times \text { haplotype } \mathrm{A} \times(\text { node }= \\
5.06311)\end{array}$ & 0.0392307 & 0.01487 & 2.77 & 0.0062 \\
\hline
\end{tabular}

\footnotetext{
${ }^{a}$ Bold values indicate statistical significance.
} 
could vary depending on the cultivar and age of the plants as well as the abiotic conditions.

Another difference identified between LsoA- and LsoB-infected plants was the progression of the bacterial abundance in the plant. Both pathogens could be detected on the top tier of the plant 2 weeks after infestation. Interestingly, early in the infection, LsoA abundance increased faster in the plant but, after 3 weeks, LsoB was more abundant than LsoA. Previously, we found that ' $\mathrm{Ca}$. L. solanacearum' distribution and movement within the plant followed a source-to-sink pattern, with higher abundance in the top of the plant (Levy et al. 2011). Although a similar distribution was found for LsoB at weeks 3, 5, and 7 after infection, LsoA-infected plants only displayed this pattern 3 weeks after infection. Interestingly, at later time points, a higher ' $\mathrm{Ca}$. L. solanacearum' abundance was measured in the middle tier of the plant. This change in distribution could be the result of a disruption of phloem transport (Brodersen et al. 2014; Koh et al. 2012). Importantly, the difference between LsoA and LsoB in distribution also could have consequences for pathogen detection. Indeed, based in our previous study on potato and tomato, it was recommended to sample plants on the top tier to maximize the probability of detecting the pathogen. However, based on the current results, it appears that LsoA abundance in the top tier of the plant can be low after 5 weeks postinfection, even below detection level. It is unknown whether LsoA has a similar distribution in potato and tomato; however, this result could explain the reported difficulties to detect this bacterium in New Zealand, where only LsoA is present (Beard et al. 2013). Nevertheless, no differences in ' $\mathrm{Ca}$. L. solanacearum' abundance in roots between the ' $\mathrm{Ca}$. L. solanacearum' haplotypes were identified at weeks 3,5 , or 7 postinfection. By week 7, ' $C a$. L. solanacearum' was more abundant in the roots than in the aerial tissues, confirming previous reports of higher ' $\mathrm{Ca}$. L. solanacearum' abundance in the roots of ' $C a$. L. solanacearum'-infected potato plants (Li et al. 2009).

Understanding the movement and abundance of ' $\mathrm{Ca}$. L. solanacearum' in plants supports diagnostic efforts. In this study, we showed that LsoA and LsoB do not have the same effect or the same distribution in tomato plants; therefore, different strategies are necessary to optimize diagnostics. Furthermore, comparison of translocation patterns between haplotypes (Cooper et al. 2015) will contribute to a better understanding of disease tolerance and mechanisms of
plant-'Ca. L. solanacearum' interaction (Levy et al. 2017). The study of the interaction between different bacterial haplotypes and the eukaryotic hosts can yield key information on the infectious mechanisms used by these microorganisms. The present study indicates that LsoA and LsoB might deploy different mechanisms to infect tomato plants or that tomato plants induce different defense responses upon LsoA or LsoB infection. These differences result in the development of symptoms that include changes in plant development. ' $\mathrm{Ca}$. L. solanacearum' is also a pathogen of the psyllid vector (Nachappa et al. $2012 b$ ) and LsoB is more pathogenic to the psyllid vector than LsoA, lowering both the number of eggs produced by females and the nymphal survival (Yao et al. 2016). Here, we showed that LsoB appears to be more pathogenic to tomato than LsoA. The genomic sequences of three different ' $\mathrm{Ca}$. L. solanacearum' haplotypes are now available (Lin et al. 2011; Thompson et al. 2015; J. H. Wang et al. 2017; Zheng et al. 2014) and revealed that LsoA and LsoB are very similar, and they differ in a limited number of genes (Ravindran et al. 2018). The differences are mostly hypothetical proteins and members
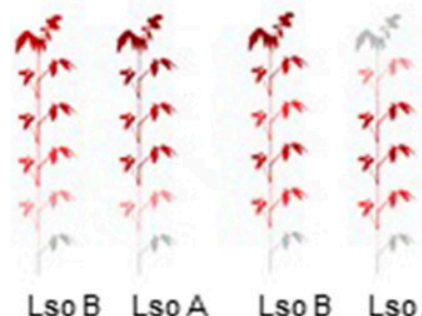

So B Lso A

Lso B Lso A

Week 3

Week 5

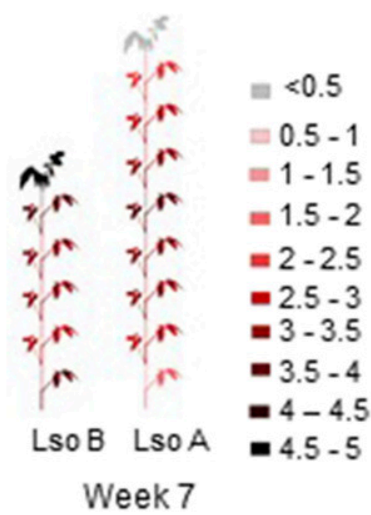

Fig. 4. Heatmap representing the progression of 'Candidatus Liberibacter solanacearum' haplotype LsoA and LsoB abundance in tomato plants at weeks 3,5 , and 7 postinfection. Shading represent ' $\mathrm{Ca}$. L. solanacearum' abundance based on $\log _{10}$ transformation of the bacterial abundance obtained by quantitative polymerase chain reaction using the standard curve method.

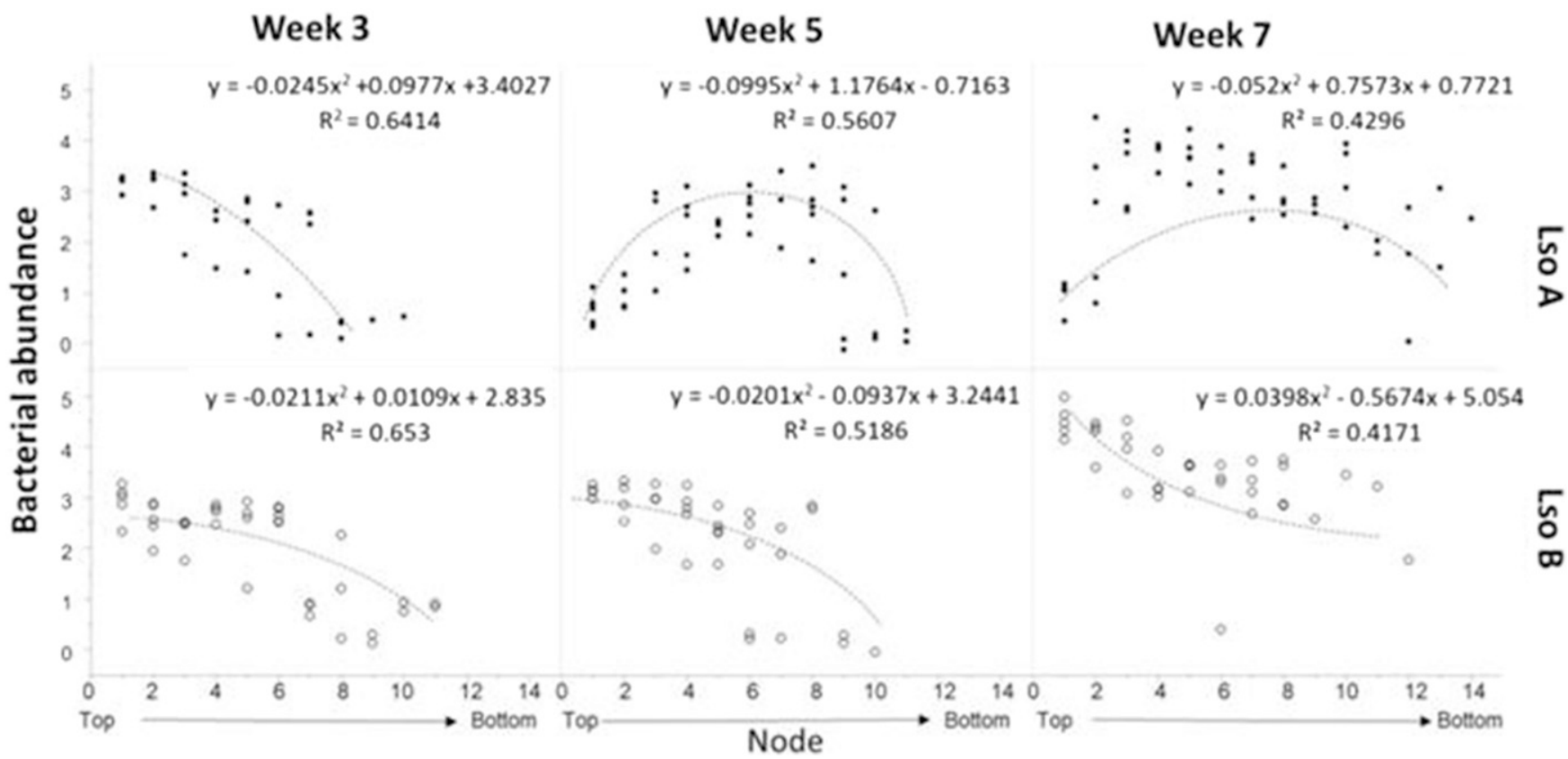

Fig. 3. Goodness-of-fit models comparing 'Candidatus Liberibacter solanacearum' abundance against node number. Graphs are structured by week (weeks 3, 5, and 7) and 'Ca. L. solanacearum' haplotype (LsoA and LsoB). Nodes were counted from top to bottom ( $\mathrm{x}$-axis), 'Ca. $\mathrm{L}$. solanacearum' abundance was calculated using the standard curve method and log-transformed (y-axis). 


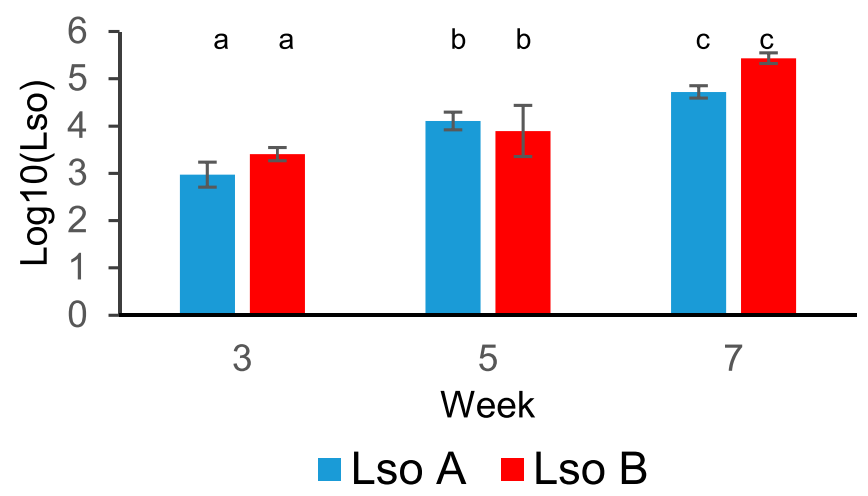

Fig. 5. 'Candidatus Liberibacter solanacearum' haplotype LsoA and LsoB abundance in roots of tomato plants 3,5 , and 7 weeks after infection. Letters indicate statistical differences between samples at $P<0.05$ using Tukey's honestly significant difference test. ' $\mathrm{Ca}$. L. solanacearum' abundance was calculated using the standard curve method and log-transformed (y-axis).

of restriction-modification systems which provide defenses against foreign DNA (bacteriophages). Although presently only haplotypes $\mathrm{A}$ and $\mathrm{B}$ have been described associated with solanaceous crops in the Americas, it is conceivable that a greater ' $\mathrm{Ca}$. L. solanacearum' genetic diversity exists. For instance, at least three LsoA strains were sequenced: HenneA $(1,211,361$ bp in length and containing 845 predicted coding sequences [CDS]) (Thompson et al. 2015), strain R1 (1,204,257 bp in length and containing 911 predicted CDS), and LsoNZ1 $(1,312,416$ bp in length and containing 1,236 predicted CDS). The identification and characterization of additional ' $\mathrm{Ca}$. L. solanacearum' haplotypes will help in understanding the molecular basis of Liberibacter pathogenicity; and the biological system composed of tomato, ' $\mathrm{Ca}$. L. solanacearum', and psyllids is well suited to identify Liberibacter virulence factors (Ibanez and Tamborindeguy 2016; Ibanez et al. 2014; Nachappa et al. 2012a). Future studies should be aimed at understanding the involvement of ' $\mathrm{Ca}$. L. solanacearum' genes that are unique to each haplotype in plant and insect infection and how they correlate with the observed differences in pathogenicity or distribution within tomato plants.

In conclusion, the present study discovered differences in pathogenicity (symptoms and bacterial abundance) associated with LsoA and LsoB haplotypes in the tomato host. These differences warrant further analyses to understand the epidemiology of Liberibacter-related diseases.

\section{Acknowledgments}

We thank D. Henne for the psyllid colony and E. A. Pierson for scientific discussions.

\section{Literature Cited}

Alfaro-Fernández, A., Siverio, F., Cebrián, M. C., Villaescusa, F. J., and Font, M. I. 2012. 'Candidatus Liberibacter solanacearum' associated with Bactericera trigonica-affected carrots in the Canary Islands. Plant Dis. 96:581.

Beard, S. S., Pitman, A. R., Kraberger, S., and Scott, I. A. W. 2013. SYBR Green real-time quantitative PCR for the specific detection and quantification of 'Candidatus Liberibacter solanacearum' in field samples from New Zealand. Eur. J. Plant Pathol. 136:203-215.

Brodersen, C., Narciso, C., Reed, M., and Etxeberria, E. 2014. Phloem production in Huanglongbing-affected citrus trees. HortScience 49:59-64.

Cooper, W. R., Alcala, P. E., and Barcenas, N. M. 2015. Relationship between plant vascular architecture and within-plant distribution of 'Candidatus Liberibacter solanacearum' in potato. Am. J. Potato Res. 92:91-99.

Crosslin, J. M., Munyaneza, J. E., Brown, J. K., and Liefting, L. W. 2010. Potato zebra chip disease: A phytopathological tale. Online publication. Plant Health Prog. doi.org/10.1094/PHP-2010-0317-01-RV

Glynn, J. M., Islam, M., Bai, Y., Lan, S., Wen, A., Gudmestad, N. C., Civerolo, E. L., and Lin, H. 2012. Multilocus sequence typing of 'Candidatus Liberibacter solanacearum' isolates from North America and New Zealand. J. Plant Pathol. 94:223-228.

Haapalainen, M. 2014. Biology and epidemics of Candidatus Liberibacter species, psyllid-transmitted plant-pathogenic bacteria. Ann. Appl. Biol. 165:172-198.
Ibanez, F., Levy, J., and Tamborindeguy, C. 2014. Transcriptome analysis of "Candidatus Liberibacter solanacearum" in its psyllid vector, Bactericera cockerelli. PLoS One 9:e100955.

Ibanez, F., and Tamborindeguy, C. 2016. Selection of reference genes for expression analysis in the potato psyllid, Bactericera cockerelli. Insect Mol Biol. 25:227-238

Koh, E.-J., Zhou, L., Williams, D. S., Park, J., Ding, N., Duan, Y.-P., and Kang, B.-H. 2012. Callose deposition in the phloem plasmodesmata and inhibition of phloem transport in citrus leaves infected with "Candidatus Liberibacter asiaticus". Protoplasma 249:687-697.

Levy, J., Ravindran, A., Gross, D., Tamborindeguy, C., and Pierson, E. 2011. Translocation of "Candidatus Liberibacter solanacearum", the zebra chip pathogen, in potato and tomato. Phytopathology 101:1285-1291.

Levy, J., and Tamborindeguy, C. 2014. Solanum habrochaites, a potential source of resistance against Bactericera cockerelli (Hemiptera: Triozidae) and “Candidatus Liberibacter solanacearum”. J. Econ. Entomol. 107:1187-1193.

Levy, J. G., Mendoza, A., Miller, J. C., Jr., Tamborindeguy, C., and Pierson, E. A 2017. Global gene expression in two potato cultivars in response to 'Candidatus Liberibacter solanacearum' infection. BMC Genomics 18:960.

Lévy, J. G., Scheuring, D. C., Koym, J. W., Henne, D. C., Tamborindeguy, C., Pierson, E., and Miller, J. C., Jr. 2015. Investigations on putative zebra chip tolerant potato selections. Am. J. Potato Res. 92:417-425.

Li, W. B., Abad, J. A., French-Monar, R. D., Rascoe, J., Wen, A. M., Gudmestad, N. C., Secor, G. A., Lee, I. M., Duan, Y. P., and Levy, L. 2009. Multiplex realtime PCR for detection, identification and quantification of 'Candidatus Liberibacter solanacearum' in potato plants with zebra chip. J. Microbiol. Methods 78:59-65

Li, X., Nie, J., Arsenault, H., Hammill, D. L., Xu, H., and De Boer, S. H. 2013 Tomato can be a latent carrier of 'Candidatus Liberibacter solanacearum', the causal agent of potato zebra chip disease. Bull. OEPP/EPPO Bull. 43:229-237.

Liefting, L. W., Perez-Egusquiza, Z. C., Clover, G. R. G., and Anderson, J. A. D. 2008. A new 'Candidatus Liberibacter' species in Solanum tuberosum in New Zealand. Plant Dis. 92:1474.

Liefting, L. W., Southerland, P. W., Ward, L. I., Paice, K. L., Weir, B. S., and Clover, G. R. G. 2009. A new "Candidatus Liberibacter" species associated with diseases of solanaceous crops. Plant Dis. 93:208-214.

Lin, H., Islam, M., Bai, Y., Wen, A., Lan, S., Gudmestad, N., and Civerolo, E. 2012. Genetic diversity of 'Candidatus Liberibacter solanacearum' strains in the United States and Mexico revealed by simple sequence repeat markers. Eur. J. Plant Pathol. 132:297-308.

Lin, H., Lou, B., Glynn, J. M., Doddapaneni, H., Civerolo, E. L., Chen, C., Duan, Y., Zhou, L., and Vahling, C. M. 2011. The complete genome sequence of 'Candidatus Liberibacter solanacearum', the bacterium associated with potato Zebra Chip disease. PLoS One 6:e19135.

Munyaneza, J. E., Crosslin, J. M., and Upton, J. E. 2007. Association of Bactericera cockerelli (Homoptera: Psyllidae) with "zebra chip," a new potato disease in southwestern United States and Mexico. J. Econ. Entomol. 100: 656-663.

Munyaneza, J. E., Fisher, T. W., Sengoda, V. G., Garczynski, S. F., Nissinen, A., and Lemmetty, A. 2010. First report of "Candidatus Liberibacter solanacearum" associated with psyllid-affected carrots in Europe. Plant Dis. 94:639.

Nachappa, P., Levy, J., Pierson, E., and Tamborindeguy, C. 2011. Diversity of endosymbionts in the potato psyllid, Bactericera cockerelli (Hemiptera: Triozidae), vector of zebra chip disease of potato. Curr. Microbiol. 62: 1510-1520.

Nachappa, P., Levy, J., Pierson, E., and Tamborindeguy, C. 2014. Correlation between "Candidatus Liberibacter solanacearum" infection levels and fecundity in its psyllid vector. J. Invertebr. Pathol. 115:55-61.

Nachappa, P., Levy, J., and Tamborindeguy, C. 2012a. Transcriptome analyses of Bactericera cockerelli adults in response to "Candidatus Liberibacter solanacearum" infection. Mol. Genet. Genomics 287:803-817.

Nachappa, P., Shapiro, A. A., and Tamborindeguy, C. 2012b. Effect of 'Candidatus Liberibacter solanacearum' on fitness of its vector, Bactericera cockerell (Hemiptera: Triozidae), on tomato. Phytopathology 102:41-46.

Nelson, W. R., Fisher, T. W., and Munyaneza, J. E. 2011. Haplotypes of "Candidatus Liberibacter solanacearum” suggest long-standing separation. Eur. J. Plant Pathol. 130:5-12

NPPO. 2017. First report of 'Candidatus Liberibacter solanacearum' on carrots in Israel. Online publication. EPPO Reporting Service no. 01, article 2017/020. https://gd.eppo.int/reporting/article-5988

Ravindran, A., Saenkham, P., Levy, J. G., Tamborindeguy, C., Lin, H., Gross, D., and Pierson, E. A. 2018. Characterization of the serralysin-like gene of ' $\mathrm{Ca}$. Liberibacter solanacearum' associated with potato zebra chip disease. Phytopathology 108:327-335.

Rojas-Martinez, R. I., Zavaleta-Mejia, E., Ochoa-Martinez, D. L., Alanis-Martines, I., and Garcia-Tapia, F. 2016. Association of Candidatus Liberibacter solanacearum with the decline of tomato (Solanum lycopersicum L.). J. Plant Pathol. 98:191-196.

Secor, G. A. and Rivera-Varas, V. V. 2004. Emerging diseases of cultivated potato and their impact on Latin America. Rev. Latinoam. Papa 1:1-8. 
Spann, T. M., and Schumann, A. W. 2009. The role of plant nutrients in disease development with emphasis on citrus and huanglongbing. Proc. Fla. State Hortic. Soc 122:169-171.

Tahzima, R., Maes, M., Achbani, E. H., Swisher, K. D., Munyaneza, J. E., and De Jonghe, K. 2014. First report of 'Candidatus Liberibacter solanacearum' on carrot in Africa. Plant Dis. 98:1426.

Tamborindeguy, C., Huot, O. B., Ibanez, F., and Levy, J. 2017. The influence of bacteria on multi-trophic interactions among plants, psyllids, and pathogen. Insect Sci. 24:961-974.

Teresani, G. R., Bertolini, E., Alfaro-Fernández, A., Martínez, C., Tanaka, F. A. O., Kitajima, E. W., Roselló, M., Sanjuán, S., Ferrándiz, J. C., López, M. M., Cambra, M., and Font, M. I. 2014. Association of 'Candidatus Liberibacter solanacearum' with a vegetative disorder of celery in Spain and development of a real-time PCR method for its detection. Phytopathology 104:804-811.

Thompson, S. M., Johnson, C. P., Lu, A. Y., Frampton, R. A., Sullivan, K. L., Fiers, M. W. E. J., Crowhurst, R. N., Pitman, A. R., Scott, I. A. W., Wen, A., Gudmestad, N. C., and Smith, G. R. 2015. Genomes of 'Candidatus Liberibacter solanacearum' haplotype A from New Zealand and the United States suggest significant genome plasticity in the species. Phytopathology 105:863-871.

Wang, J. H., Haapalainen, M., Schott, T., Thompson, S. M., Smith, G. R., Nissinen, A. I., and Pirhonen, M. 2017. Genomic sequence of 'Candidatus
Liberibacter solanacearum' haplotype $\mathrm{C}$ and its comparison with haplotype A and B genomes. PLoS One 12:e0171531.

Wang, N., Pierson, E. A., Setubal, J. C., Xu, J., Levy, J. G., Zhang, Y. Z., Li, J. Y., Rangel, L. T., and Martins, J. 2017. The Candidatus Liberibacter-host interface: Insights into pathogenesis mechanisms and disease control. Annu. Rev. Phytopathol. 55:451-482.

Wen, A., Mallik, I., Alvarado, V. Y., Pasche, J. S., Wang, X., Li, W., Levy, L., Lin, H., Scholthof, H. B., Mirkov, T. E., Rush, C. M., and Gudmestad, N. C. 2009 Detection, distribution, and genetic variability of 'Candidatus Liberibacter' species associated with zebra complex disease of potato in North America. Plant Dis. 93:1102-1115.

Wen, A. M., Johnson, C., and Gudmestad, N. C. 2013. Development of a PCR assay for the rapid detection and differentiation of 'Candidatus Liberibacter solanacearum' haplotypes and their spatiotemporal distribution in the United States. Am. J. Potato Res. 90:229-236.

Yao, J., Saenkham, P., Levy, J., Ibanez, F., Noroy, C., Mendoza, A., Huot, O., Meyer, D. F., and Tamborindeguy, C. 2016. Interactions 'Candidatus Liberibacter solanacearum'-Bactericera cockerelli: Haplotype effect on vector fitness and gene expression analyses. Front. Cell. Infect. Microbiol. 6:62.

Zheng, Z., Clark, N., Keremane, M., Lee, R., Wallis, C., Deng, X., and Chen, J. 2014. Whole-genome sequence of "Candidatus Liberibacter solanacearum" strain R1 from California. Genome Announc. 2:e01353-14 